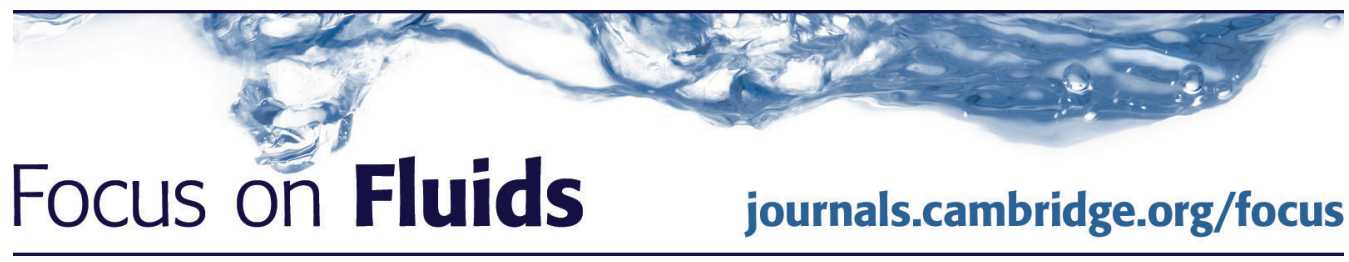

\title{
Canonical wall-bounded flows: how do they differ?
}

\author{
Alexander J. Smits ${ }^{1,2, \dagger}$ \\ ${ }^{1}$ Department of Mechanical and Aerospace Engineering, \\ Princeton University, Princeton, NJ 08544, USA \\ ${ }^{2}$ Department of Mechanical and Aerospace Engineering, \\ Monash University, Melbourne, Australia
}

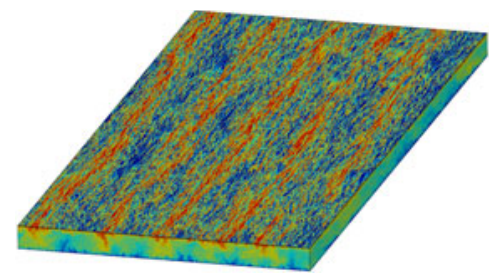

Orlandi et al. (J. Fluid Mech., vol. 770, 2015, pp. 424-441) present direct numerical simulations over a very wide Reynolds number range for plane Couette and Poiseuille flows. The results reveal new information on the abrupt nature of transition in these flows, and the comparisons between Couette and Poiseuille flows help to provide a clearer picture of Reynolds number trends, especially with regard to inner/outer layer interactions. The stress distributions give strong support to Townsend's attached eddy hypothesis, particularly for the wall-parallel component where there has been little experimental data available. The results pose some intriguing questions regarding the reconciliation of the present results with data at higher Reynolds numbers in different canonical flows.

Key words: boundary layer structure, turbulence simulation

\section{Introduction}

For practical purposes, we are especially interested in turbulent flows in proximity to a boundary, often called wall-bounded flows. To make progress in understanding such flows, researchers have often focused on a small number of canonical flows that are subject to relatively simple boundary conditions: developing flows such as zero pressure gradient boundary layers, and a variety of fully developed flows such as Couette flows and Poiseuille flows.

Orlandi, Bernardini \& Pirozzoli (2015) present a comprehensive set of direct numerical simulations (DNS) for two canonical turbulent flows: plane Poiseuille flow (where the flow is driven by a pressure gradient), and plane Couette flow (where the flow is driven by a moving boundary). In both cases, the net acceleration is zero, and the flow is steady and two-dimensional in the mean. For Poiseuille flow, the Navier-Stokes equation reduces to

$$
-\overline{u^{\prime} v^{\prime}}+v(\mathrm{~d} u / \mathrm{d} y)=u_{\tau}^{2}(1-y / h)
$$

where $\tau_{w}\left(=\rho u_{\tau}^{2}\right)$ is the wall stress given by $-h \mathrm{~d} p_{w} / \mathrm{d} x$. Here, $p_{w}$ is the wall pressure, $u^{\prime}$ and $v^{\prime}$ are the streamwise and wall-normal velocity fluctuations respectively, $y$ is the

$\dagger$ Email address for correspondence: asmits@princeton.edu 
wall-normal distance, $h$ is the channel half-width, $u$ is the mean velocity, and $\rho$ and $v$ are the fluid density and kinematic viscosity. We see that in Poiseuille flow, the total stress, that is, the sum of the turbulent and viscous stresses, varies linearly across the flow. Couette flow is even simpler, in that the total stress is constant.

Equation (1.1) also illustrates the essential character of all wall-bounded turbulent flows, in that the relative influence of the viscous and turbulent stresses depends on the distance from the wall. In the region near the wall (the 'inner region'), the turbulent velocity fluctuations go to zero to satisfy the no-slip condition, and the mean velocity gradients are high so that the viscous stress dominates. Moving away from the wall, the turbulence level rises quickly while the mean velocity gradient decreases, and far from the wall in the 'outer region' the viscous stress are negligible compared to the turbulent stresses.

One of the most interesting questions in studying wall-bounded flows is the level of interaction between the inner and outer flow. The ratio of outer length scale $h$ to the inner length scale $v / u_{\tau}$ represents the 'friction' Reynolds number $R e_{\tau}=h u_{\tau} / v$. We expect that as $R e_{\tau}$ increases, the distinction between the inner scaling and the outer scaling will sharpen, but will there be a point where the inner and outer layer become independent?

\section{Overview}

The DNS results presented by Orlandi et al. (2015) cover Reynolds numbers from the laminar regime into the turbulent regime, and the highest Reynolds numbers are among the highest values achieved so far in DNS. Therefore they present an ideal platform for examining Reynolds number effects using a self-consistent data base. Orlandi et al. also offer an evaluation of the effects of boundary conditions by comparing Poiseuille flow and Couette flow. Most previous studies have focused on plane Poiseuille flow (for some recent examples, see Jiménez \& Hoyas 2008 and Lozano-Durán \& Jiménez 2014), and plane Couette flows have not been as widely studied, probably because they are difficult to reproduce experimentally. Orlandi et al. make a persuasive case that Couette flow can help to elucidate the effects of Reynolds number, especially for inner/outer interactions, because its core flow is quite different from that of Poiseuille flow. Given that DNS is supplanting low-Reynolds-number experiments, at least for fundamental studies such as this one, the lack of experimental validation is perhaps not such a pressing concern as it used to be. That is, a detailed study of plane Couette flow is overdue, and the present contribution is therefore very welcome.

A particularly important aspect of these wide-ranging computations is that they were performed with a consistent numerical method; Reynolds number effects are generally rather subtle, and so it is important to understand the effects of spatial resolution, domain size, and time evolution. The authors are very aware of these issues, and so comparisons across Reynolds numbers and boundary conditions can be made with confidence.

Transition in both flows appears as a sharp jump in wall friction and bulk turbulent kinetic energy, that is, the kinetic energy associated with the turbulent velocity fluctuations, integrated over the bulk flow. Interestingly, for the same bulk velocity, the Couette flow has a $23 \%$ lower skin friction in the turbulent regime compared to Poiseuille flow. When the skin friction and the Reynolds number are scaled on the centreline velocity rather than the bulk velocity, however, the two flows show good collapse, and they also agree well with pipe flow data, which is an original and provocative result. 
As to the turbulent mean velocity profile, the authors do not find a convincing region of logarithmic variation for either flow, which is in accord with similar studies of channel and pipe flow. Nevertheless, the two flows show some level of similarity in that the Couette flow follows an evolution with Reynolds number similar to that of the Poiseuille flow, except that it appears to be shifted towards a higher Reynolds number. This trend is also seen in the Reynolds stress behaviour, suggesting that Reynolds number effects are generally more significant in Couette flow compared to Poiseuille flow.

At a sufficiently high Reynolds number, Townsend's attached eddy hypothesis suggests that the streamwise and spanwise stresses should display a logarithmic variation in the overlap region. Experiments have revealed this behaviour for the streamwise turbulence intensity when $R e_{\tau}>10000$ (Hultmark et al. 2012; Marusic et al. 2013). This behaviour is not seen here for the streamwise component in Couette flow, but a short region is found for Poiseuille flow at the highest Reynolds number. What is particularly interesting, however, is that the authors find a convincing region of logarithmic variation in the spanwise component, something that was seen only in a nascent sense at lower Reynolds numbers (Jiménez \& Hoyas 2008). The Couette flow shows a larger region of fit, consistent with a faster evolution with Reynolds number. There is a lack of experimental data on the spanwise component (it is rather difficult to measure), and so the numerical results are especially valuable here.

The behaviour of the streamwise turbulence intensity is important in at least two respects. First, the peak in $u^{\prime 2^{+}}\left(=\overline{u^{\prime 2}} / u_{\tau}^{2}\right)$ in the near-wall region is a measure of the inner/outer interaction: a peak that is invariant in inner units (in location and magnitude) would signal little or no effect of the outer scale turbulence on the near-wall region. The results given here show that the peak in $u^{\prime 2^{+}}$is a relatively weak function of $R e_{\tau}$ : the mean square peak in Couette flow varies with $\log R e_{\tau}$ approximately with a slope of 0.86 , while for Poiseuille flow it has a smaller slope of about 0.5 . Second, $u^{\prime 2^{+}}$plays an important in the production of turbulent kinetic energy, in that the production takes place in the streamwise component and it is then redistributed by pressure fluctuations to the other two components. In this respect, the results shown in Orlandi et al. indicate that the production in the wall region $\left(y^{+}<50\right)$ for both flows is almost entirely independent of Reynolds number for $R e_{\tau}>180$. The outer limit for similarity increases with Reynolds number, and according to the present author is estimated to be fixed at $y / h \approx 0.15$. The premultiplied production indicates a very different distribution of the contributions to the production; since, in Couette flow, the flow is not symmetric about the centreline, there is significant energy production in the bulk flow, leading to the formation of strong streamwise structures, something that is absent in Poiseuille flow.

\section{Future}

Orlandi et al. compare Couette flows with Poiseuille flows over a wide range of Reynolds numbers, and identify many similarities between the two flows, particularly in terms of the abrupt transition behaviour, the skin friction behaviour, and the near Reynolds number independence of the mean velocity, kinetic energy production, and turbulent viscosity in the near-wall region. The differences are also striking: it is clear that in terms of the turbulent stresses Couette flow shows a stronger Reynolds number dependence than Poiseuille flows, and Couette flow appears to reach a high-Reynoldsnumber state more quickly than Poiseuille flow while sharing the same general trends.

Given that these new DNS data from Couette flow are so useful, a case could be made for examining other canonical flows in more detail using DNS, and 
moving beyond plane Poiseuille flow. For pipe flow, for example, there are numerous experimental data sets available but very few simulations (a notable exception being Wu, Baltzer \& Adrian 2012). Couette flows with pressure gradients might also be a logical step, especially since the pressure gradient can be controlled in a way that is not possible in Poiseuille flow. Earlier work by the same authors provides a ready-made platform for this venture (Orlandi, Bernardini \& Pirozzoli 2011).

A particularly provocative result from Orlandi et al. is that the slope of the logarithmic stress variations is the same for $u^{\prime 2^{+}}$and $w^{\prime 2^{+}}$, and an argument is made that they are the same in Poiseuille and Couette flows. The value found here, however, is 0.386 , which is smaller than the value of 0.5 found for $w^{\prime 2^{+}}$by Jiménez \& Hoyas (2008), and very much smaller than the value of 1.25 found for $u^{\prime 2^{+}}$in high-Reynolds-number boundary layer and pipe flows (Marusic et al. 2013), which raises some interesting questions on the evolution of this slope with Reynolds number, and/or flow geometry, and also the fitting range used to find the slope. In addition, the peak in $u^{\prime 2^{+}}$in Poiseuille flow varies with $\log R e_{\tau}$ approximately with a slope of 0.5 , whereas Lozano-Durán \& Jiménez (2014) found a slope of 0.642 for nominally the same Poiseuille flow. This comparison raises a larger question, namely the apparent difficulty of determining this peak. Experiments and computations disagree considerably on its variation with Reynolds number, and how flow-dependent it may be. It would seem that, given the important role of $u^{\prime 2^{+}}$in the production of turbulent kinetic energy, and as a measure on the level of inner/outer interaction, we need to resolve its behaviour with more certainty in future work.

The work by Orlandi et al. also raises some new questions that perhaps can only be answered by analysis. For example, it is apparent that the spanwise component exhibits logarithmic variation at a considerably lower Reynolds number than the streamwise component. Why should this be so, and how does it impact our understanding of the attached eddy hypothesis? Also, Orlandi et al. show that the mean velocity variations in the core of Couette and Poiseuille flow appear to be described well by a linear and parabolic profile, respectively. Again, why should this be so, and does this behaviour also happen in pipe flow? What are the implications for wake formulations? We look forward to seeing further work in this fascinating field.

\section{References}

Hultmark, M., Vallikivi, M., Bailey, S. C. C. \& Smits, A. J. 2012 Turbulent pipe flow at extreme Reynolds numbers. Phys. Rev. Lett. 108 (9), 1-5.

JimÉnEZ, J. \& HoYAS, S. 2008 Turbulent fluctuations above the buffer layer of wall-bounded flows. J. Fluid Mech. 611, 215-236.

LozAno-DurÁn, A. \& JimÉnEZ, J. 2014 Effect of the computational domain on direct simulations of turbulent channels up to $R e_{\tau}=4200$. Phys. Fluids 26, 011702.

Marusic, I., Monty, J. P., Hultmark, M. \& Smits, A. J. 2013 On the logarithmic region in wall turbulence. J. Fluid Mech. 716, R3.

Orlandi, P., Bernardini, M. \& Pirozzoli, S. 2011 Large-scale organization and inner-outer layer interactions in turbulent Couette-Poiseuille flows. J. Fluid Mech. 680, 534-563.

Orlandi, P., Bernardini, M. \& Pirozzoli, S. 2015 Poiseuille and Couette flows in the transitional and fully turbulent regime. J. Fluid Mech. 770, 424-441.

Wu, X., Baltzer, J. R. \& Adrian, R. J. 2012 Direct numerical simulation of a 30R long turbulent pipe flow at $R^{+}=685$ : large- and very large-scale motions. J. Fluid Mech. 698, 235-281. 